ARTICLE

\title{
Bacteria can mobilize nematode-trapping fungi to kill nematodes
}

Xin Wang ${ }^{1, \star}$, Guo-Hong $\mathrm{Li}^{1, \star}$, Cheng-Gang Zou ${ }^{1, \star}$, Xing-Lai Ji ${ }^{1}$, Tong Liu ${ }^{1}$, Pei-Ji Zhao ${ }^{2}$, Lian-Ming Liang ${ }^{1}$, Jian-Ping $X u^{1,3}$, Zhi-Qiang $A n^{4}$, Xi Zheng ${ }^{1}$, Yue-Ke Qin ${ }^{1}$, Meng-Qing Tian ${ }^{1}$, You-Yao Xu', Yi-Cheng Ma', Ze-Fen Yư ${ }^{1}$ Xiao-Wei Huang ${ }^{1}$, Shu-Qun Liu${ }^{1}$, Xue-Mei Niu${ }^{1}$, Jin-Kui Yang${ }^{1}$, Ying Huang ${ }^{1} \&$ Ke-Qin Zhang ${ }^{1}$

In their natural habitat, bacteria are consumed by bacterivorous nematodes; however, they are not simply passive preys. Here we report a defensive mechanism used by certain bacteria to mobilize nematode-trapping fungi to kill nematodes. These bacteria release urea, which triggers a lifestyle switch in the fungus Arthrobotrys oligospora from saprophytic to nematodepredatory form; this predacious form is characterized by formation of specialized cellular structures or 'traps'. The bacteria significantly promote the elimination of nematodes by A. oligospora. Disruption of genes involved in urea transport and metabolism in A. oligospora abolishes the urea-induced trap formation. Furthermore, the urea metabolite ammonia functions as a signal molecule in the fungus to initiate the lifestyle switch to form trap structures. Our findings highlight the importance of multiple predator-prey interactions in prey defense mechanisms.

\footnotetext{
${ }^{1}$ Laboratory for Conservation and Utilization of Bio-Resources, Yunnan University, Kunming, Yunnan 650091, China. ${ }^{2}$ State Key Laboratory of Phytochemistry and Plant Resources in West China, Kunming Institute of Botany, Chinese Academy of Sciences, Kunming, Yunnan 650201, China. ${ }^{3}$ Department of Biology, McMaster University, Hamilton, Ontario, Canada L8S 4K1. ${ }^{4}$ Texas Therapeutics Institute, The Brown Foundation Institute of Molecular Medicine, University of Texas Health Science Center, Texas 77030, USA. ${ }^{*}$ These authors contributed equally to this work. Correspondence and requests for materials should be addressed to K.-Q.Z. (email: kqzhang@ynu.edu.cn).
} 
n natural ecosystems, bacterial communities play an important role in nutrient cycling, primary production and decomposition $^{1}$. Bacteria are also heavily consumed by bacterivorous nematodes that are present in compost, soil and aquatic systems $^{1,2}$. Thus, aside from competing for nutrients among themselves, bacteria experience significant predation pressure from nematodes. To counter predatory attacks from the metazoan enemies, bacteria have evolved a variety of defense mechanisms ${ }^{3}$. First, many bacteria can produce and secrete toxins. For instance, the bacterium Pseudomonas fluorescens secretes secondary metabolites to repel nematodes ${ }^{4}$, whereas $P$. aeruginosa produces phenazine compounds and cyanide to kill nematodes ${ }^{5,6}$. Second, the secretion of extracellular proteases contributes to the nematode toxicity of the bacteria Vibrio cholerae and Bacillus nematocida against nematodes ${ }^{7,8}$. Third, morphological adaptations by bacteria are also a common defense mechanism. For example, biofilm formation by the bacterium Yersinia pestis can prevent or reduce ingestion by nematodes (and other predators $)^{9,10}$. These specific strategies provide certain bacteria an advantage against their nematode predators.

These bacterial defense mechanisms in turn create selective pressure on nematodes, resulting in predator-prey arms race. For example, nematodes have evolved to avoid the toxic/pathogenic bacteria and forage for nontoxic bacteria to support their growth and reproduction ${ }^{11}$. Currently, the most detailed data on nematode behavioral responses to bacteria have come from the model nematode Caenorhabditis elegans ${ }^{12}$. Although C. elegans has a relatively simple nervous system, its sensory neurons can detect many environmental cues. When encountering bacterial pathogens, $C$. elegans exhibits an avoidance behaviour and escapes $^{13-15}$. In the presence of nontoxic bacteria, C. elegans can recognize and hunt for them as food sources. However, it remains unclear whether counter response strategies exist in these nontoxic bacteria.

In natural environments, the nematode-trapping fungi can trap and kill nematodes. These fungi have two lifestyle phases: saprophytic and predacious ${ }^{16,17}$. In the absence of nematodes, these fungi live as saprophytes. Upon encountering nematodes, these fungi enter the predacious stage by producing specialized predatory devices to capture and kill nematodes. Earlier studies have demonstrated that nematodes produce signal substances to promote trap formation in these fungi ${ }^{18}$, but the chemical compositions of these substances remain largely unknown. A recent study has reported that ascarosides, a conserved family of signalling molecules that are widely biosynthesized among nematodes ${ }^{99}$, induce trap formation in nematodetrapping fungi ${ }^{20}$. Aside from ascarosides, diffusible compounds from cow dung have also shown to induce lifestyle transitions in the model nematode-trapping fungus Arthrobotrys oligospora, which produces three-dimensional (3D) adhesive networks ${ }^{21}$.

We hypothesized that the diffusible components from cow dung could have a bacterial origin. Here we report that urea released from bacteria triggers a lifestyle switch in the fungus A. oligospora from saprophytic to predacious stage. Ammonia, a product of urea degradation, in turn serves as a signal molecule to initiate this lifestyle switch. These findings provide a striking example of multiple predator-prey interactions, which play an important role for modulating the composition and population dynamics in nature.

\section{Results}

Bacteria in cow dung are capable of inducing trap formation. Consistent with a previous observation, we found that fresh cow dung induced trap formation in A. oligospora on water agar plates (Fig. 1a). However, autoclaved dung samples induced fewer traps than fresh samples (Supplementary Fig. 1). As bacteria constitute a significant portion of the cow dung biota, these results suggest that active metabolites from bacteria in cow dung may serve as inducers of trap formation in A. oligospora. To test this idea, we first identified the specific bacteria in cow dung that can induce trap formation in A. oligospora. The aqueous suspension of fresh dung was serially diluted by 10 -folds in sterilized water and spread-plated on nutrient Luria-Bertani broth (LB) agar. After 1 day of incubation, a variety of bacterial colonies appeared and 126 random colonies (named CD1 to CD126) were subcultured and analysed. 16S rRNA gene analysis clustered the 126 isolates into 18 genera (Supplementary Table 1). Of these isolates, supernatants from 55 isolates in LB broth medium could induce trap formation in A. oligospora. Three Stenotrophomonas maltophilia isolates (CD8, CD52 and CD103) were the most efficient inducers. In contrast, the supernatants of the remaining 71 isolates did not induce trap formation. These results indicate that some bacterial isolates obtained from cow dung can induce trap formation in A. oligospora.

Identification of urea as the trap inducer in A. oligospora. Of the 55 isolates capable of inducing trap formation in A. oligospora, three S. maltophilia isolates (CD8, CD52 and CD103) were the most efficient ones. To determine the compound(s) secreted from the bacteria that induced trap formation in A. oligospora, the extract from fermentation supernatant of S. maltophilia CD52 in LB medium was isolated by silica gel G column and Sephadex LH-20 column chromatography. A candidate compound was obtained by activity-guided isolation, and further identified as urea with mass spectrometry, nuclear magnetic resonance and elemental analysis (Supplementary Fig. 2a-c; Fig. 1b). Urea was also found in the fermentation supernatant of $S$. maltophilia CD52 in a low-nutrient minimal medium (MM; Fig. 1b). These results suggest that urea is produced by $S$. maltophilia CD52 in both nutrient-rich and -deficient medium. Furthermore, urea was detected in the fermentation supernatants from the other 54 isolates that showed trap-inducing activity, but not from the remaining 71 isolates (Supplementary Table 1).

The observation that urea secreted by bacteria could induce fungal trap formation was further confirmed by analysing commercial ultrapure grade urea on water agar plates. After 72$\mathrm{h}$ inoculation, urea significantly induced trap formation in $A$. oligospora in a dose-dependent manner, reaching maximum induction of trap formation at $300 \mathrm{mgl}^{-1}$ (Fig. 1c). Interestingly, at the high concentration of $1,500 \mathrm{mgl}^{-1}$, urea completely abolished the trap formation (Fig. 1c). Recently, Hsueh et al. ${ }^{20}$ have demonstrated that ascarosides produced by $C$. elegans can induce trap formation in nematode-trapping fungi. However, trap induction by ascarosides was completely suppressed in a medium containing $0.5 \%\left(5,000 \mathrm{mgl}^{-1}\right)$ of $\left(\mathrm{NH}_{4}\right)_{2} \mathrm{SO}_{4}$. Thus, the results from our study and that of Hsueh et al. ${ }^{20}$ are consistent with the hypothesis that trap formation is inhibited under nitrogen-rich conditions. Next, we determined whether urea existed in the exudation of cow dung and found that the concentration of urea in exudation of cow dung was $151 \mathrm{mgl}^{-1}$. Furthermore, the exudation of cow dung significantly induced the trap formation in A. oligospora (Supplementary Fig. 3).

In bacteria, urea is generated mainly through arginine catabolism in which arginase converts arginine into ornithine and urea in the last step of the urea cycle ${ }^{22}$. We randomly selected three urea-producing (CD52, CD82 and CD101) and three nonurea-producing bacteria (CD1, CD93 and CD102) that were identified at the species level, and tested whether putative $\operatorname{arcA}$ genes encoding arginases exist in the genomes of these bacteria and whether they are expressed. We amplified putative $\operatorname{arcA}$ 
a

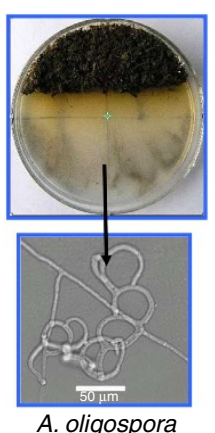

b

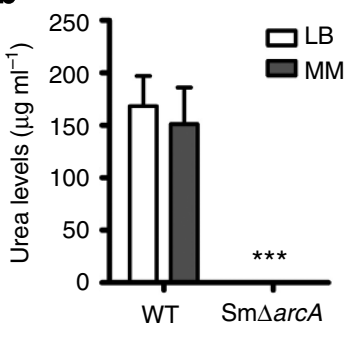

C

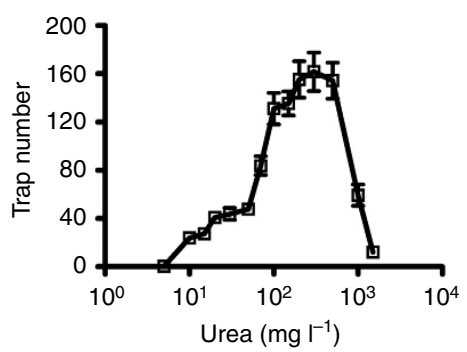

d

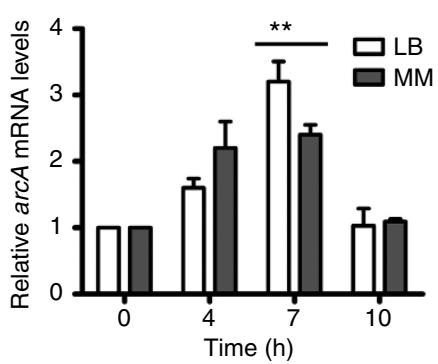

e

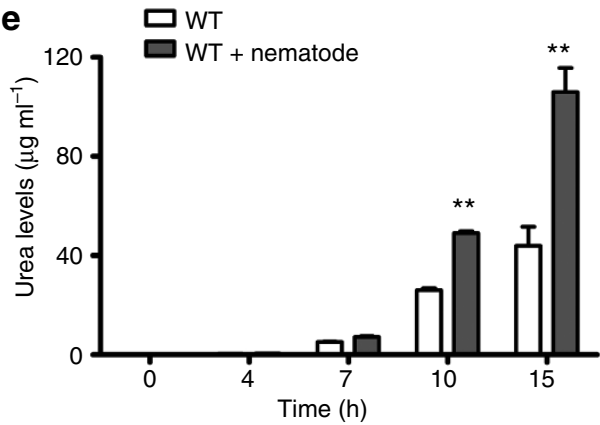

f

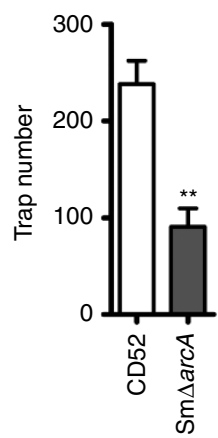

Figure 1 | Urea produced by bacteria in cow dung functions as a signal molecule to induce trap formation in A. oligospora. (a) Trap formation in A. oligospora in the vicinity of cow dung. Cow dung (brown area in the bottom of the plate) was placed on a water agar plate. After conidia of A. oligospora were added at position $2 \mathrm{~cm}$ away from dung, traps were produced (arrow). (b) Deletion of $\operatorname{arcA}$ completely abolished the production of urea in $S$. maltophila CD52 in LB medium and MM, respectively. Data are expressed as mean \pm s.d. of three independent experiments. ${ }^{\star \star \star} P<0.001$ versus CD52 ( $t$-test). (c) Effect of urea concentration on trap formation in A. oligospora at $72 \mathrm{~h}$. (d) Expression of $\operatorname{arcA}$ in $\mathrm{S}$. maltophila was induced by the addition of nematodes in LB medium and MM. Data are expressed as mean \pm s.d. of three independent experiments. ${ }^{\star \star} P<0.01$ versus control $(0 \mathrm{~h}$; $t$-test). (e) The urea levels in the supernatant in S. maltophila were elevated by nematodes. Data are expressed as mean \pm s.d. of three independent experiments. ${ }^{\star \star} P<0.01$ versus wild-type (WT) only ( $t$-test). (f) Deletion of $\operatorname{arcA}$ significantly suppressed S. maltophila-mediated trap formation in A. oligospora. Data are expressed as mean \pm s.d. of three independent experiments. ${ }^{\star \star} P<0.01$ versus CD52 ( $t$-test).

genes from the genomic DNA and mRNA, respectively, by PCR using two degenerate primers. The amplified fragments were obtained from the genomic DNA of all the bacteria (Supplementary Fig. 4). DNA sequencing demonstrated that these fragments contain the $\operatorname{arcA}$ genes. In contrast, the amplified fragments were only obtained from the mRNA of the three ureaproducing bacteria, but not of the non-urea-producing bacteria (Supplementary Fig. 4). The results suggest that the $\operatorname{arcA}$ genes might be induced under certain conditions in these non-ureaproducing bacteria.

To determine whether arginase was required for urea production in S. maltophila CD52, we deleted the $\operatorname{arcA}$ gene in this strain (Supplementary Fig. 5a,b). As expected, no urea was detected in the fermentation supernatant from the SM $\Delta a r c A$ mutant (Fig. 1b). We found that the expression of $\operatorname{arcA}$ in $S$. maltophila CD52 was significantly upregulated at $7 \mathrm{~h}$ after exposure to the nematode C. elegans in both LB medium and MM (Fig. 1d). Consistently, the urea levels in the supernatant were also markedly increased at $10 \mathrm{~h}$ after exposure to C. elegans in the LB medium (Fig. 1e). Finally, the deletion of $\operatorname{arcA}$ significantly suppressed the ability of S. maltophila CD52 to induce trap formation in A. oligospora (Fig. 1f). These results suggest that urea produced from bacteria induces trap formation in A. oligospora.

To investigate whether urea could induce trap formation in other nematode-trapping fungi, we tested urea responsiveness in additional species of the fungal family Orbiliaceae, Ascomycota. These fungi can produce a variety of trap structures including $3 \mathrm{D}$ adhesive networks, adhesive knobs, adhesive columns and constricting rings (Supplementary Table 2). We found that urea induced trap structures in 7 of the 31 species under the tested conditions (Fig. 2). For the remaining 24 species, it is likely that they respond to other signal molecules yet to be identified. The fact that the trap structures produced by these species cover most of the trap types suggests that urea responsiveness is common, but not conserved, among nematode-trapping fungi.

Urea uptake is crucial for trap formation in A. oligospora. A number of transporters from fungi with high specificity for urea have been reported ${ }^{23-25}$. From the genome of $A$. oligospora ATCC24927 (ref. 17), we identified two putative urea transporter genes utp79 and utp215 (AOL_S00079g183r and AOL_s00215g323r). Phylogenetic analyses showed that these two urea transporters from A. oligospora were clustered into two separate groups (Supplementary Fig. 6). To determine whether Utp79 and Utp215 are the active urea transporters in A. oligospora, we deleted the two genes by homologous recombination (Supplementary Fig. $7 \mathrm{a}-\mathrm{c}$ ). Colony growth of the Ao $\Delta$ utp215 mutant strain was similar to that of the wild-type strain on potato dextrose agar (PDA) or corn meal agar (CMA), whereas the Ao $\Delta u t p 79$ mutant strain grew slower than the wildtype strain on PDA, but not on CMA (Supplementary Fig. 8). Next, we measured urea uptake using $\left[{ }^{13} \mathrm{C},{ }^{15} \mathrm{~N}_{2}\right]$-urea as a tracer. The $\left[{ }^{13} \mathrm{C},{ }^{15} \mathrm{~N}_{2}\right]$-urea uptake was completely abolished when utp79 was disrupted, whereas the disruption of $u t p 215$ showed 
only partially impaired $\left[{ }^{13} \mathrm{C},{ }^{15} \mathrm{~N}_{2}\right]$-urea uptake (Fig. 3a). Importantly, the disruption of utp79 completely blocked the urea-induced trap formation, whereas the trap formation was

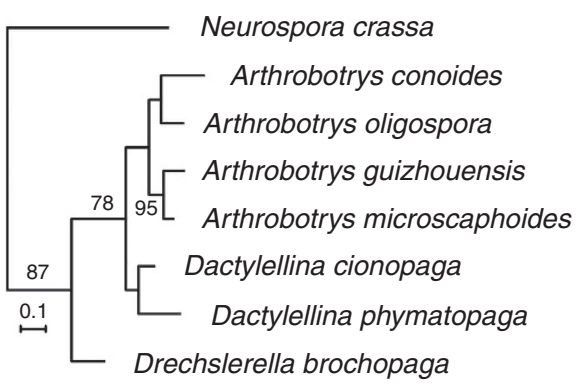

only partially suppressed in the Ao $\Delta u t p 215$ mutant (Fig. 3b). These results indicate that urea needs to be transported into fungal cells to mediate trap formation in A. oligospora, mainly through Utp79.

The urea metabolite ammonia functions downstream to induce trap formation. Generally, one urea molecule is converted to two ammonia molecules and one carbon dioxide molecule. From the genome of $A$. oligospora $^{17}$, a putative urease gene, namely ure 1 (AOL_s00080g26r), was retrieved and analysed (Supplementary Fig. 9). We deleted ure1 and found that the Aosure1 mutant strain grew slower than the wild-type strain on PDA and CMA (Supplementary Figs $7 \mathrm{~d}$ and 8 ). Deletion of ure 1 resulted in significant intracellular accumulation of $\left[{ }^{13} \mathrm{C},{ }^{15} \mathrm{~N}_{2}\right]$-urea in $A$. oligospora (Fig. 3c). However, increased extracellular urea concentration (up to $300 \mathrm{mgl}^{-1}$ ) failed to induce trap formation in the Aosure1 mutant (Fig. 3d). These data suggest that urea breakdown product(s) is crucial for the induction of trap formation.

We next investigated which of the two urea-breakdown products serves as the signal molecule to promote trap formation in A. oligospora. Unlike in Candida albicans and Cryptococus neoformans where carbon dioxide was effective at inducing morphological change 26,27 , carbon dioxide at a range of concentrations $(1,2.5$ and $5 \%)$ failed to induce trap formation in A. oligospora. In contrast, ammonia effectively elicited trap formation in the wild-type strain (Fig. 3d). Similar to the response

Figure 2 | Urea-induced morphogenesis is common in nematodetrapping fungi. Bayesian inference phylogeny of the ITS regions of nematode-trapping fungi in the family Orbiliaceae, Ascomycota. The model filamentous fungus Neurospora crassa is used as outgroup. Trapping structures are $\mathrm{AC}$, adhesive columns; $\mathrm{AK}$, adhesive knobs; $\mathrm{AN}$, adhesive networks and $\mathrm{CR}$, constricting rings.
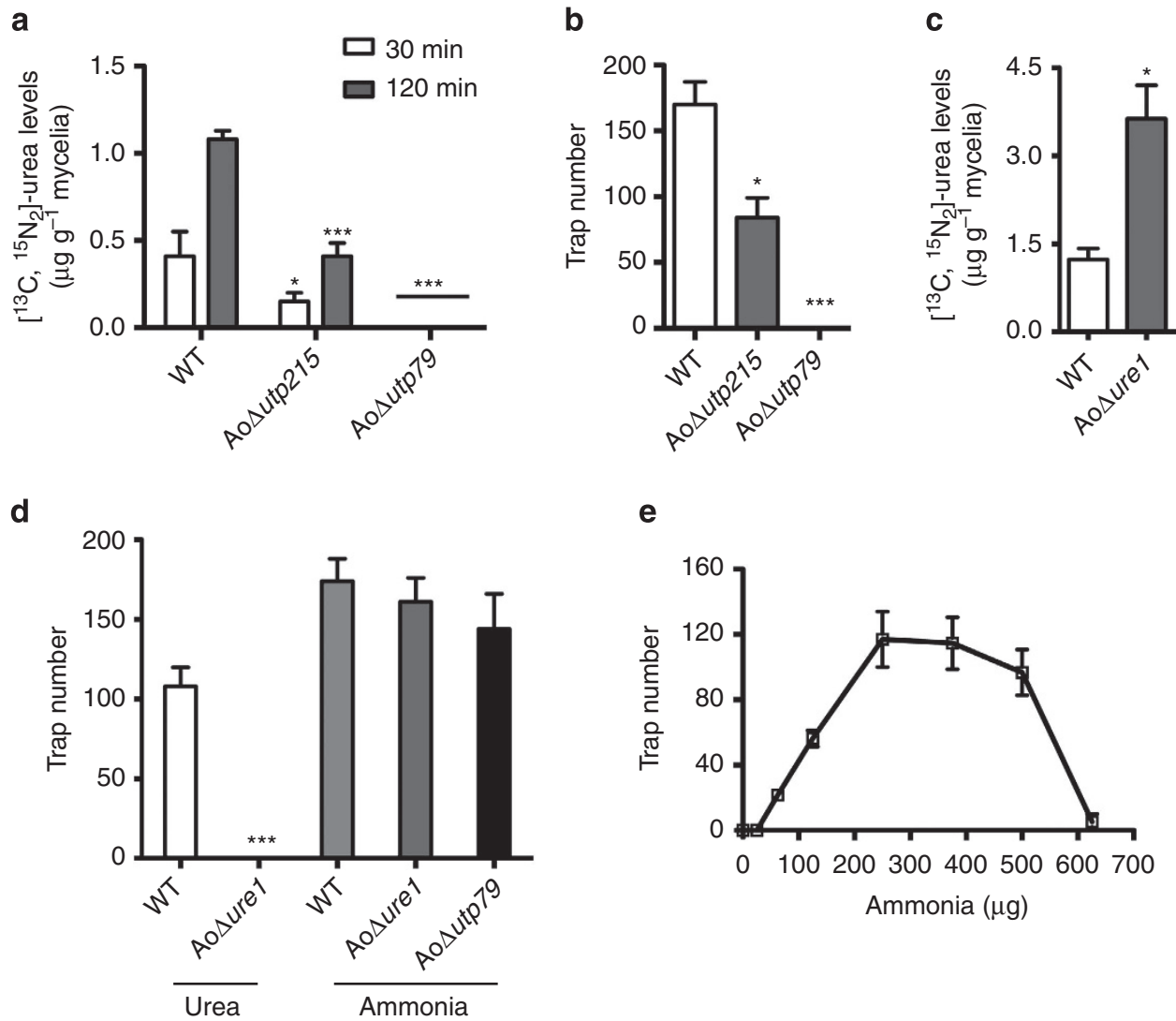

e

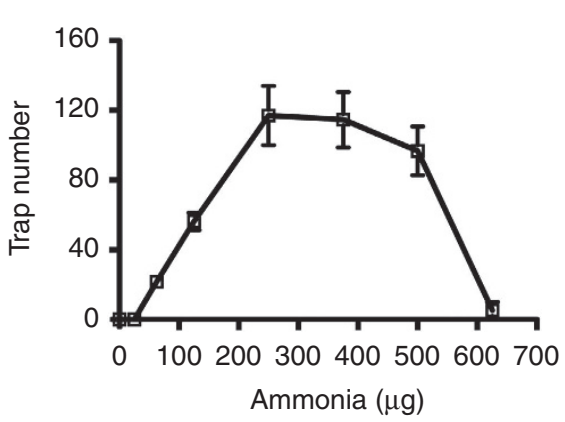

Figure 3 | The transport and decomposition of urea are required for trap formation in $\boldsymbol{A}$. oligospora. (a) $\left[{ }^{13} \mathrm{C}_{1}^{15} \mathrm{~N}_{2}\right]-$-Urea uptake was completely abolished in the Aosutp79 mutant. Disruption of utp215 only partially impaired $\left[{ }^{13} \mathrm{C}_{1}^{15} \mathrm{~N}_{2}\right]$-urea uptake in $A$. oligospora. (b) Deletion of utp 79 eliminated the urea-induced trap formation in A. oligospora. In contrast, deletion of utp215 partially inhibited urea-mediated trap formation. (c) Deletion of ure1 led to urea accumulation in A. oligospora. $\left[{ }^{13} \mathrm{C}_{1}^{15} \mathrm{~N}_{2}\right]$-urea uptake was measured in the WT and Aosure1 mutant strains. (d) Deletion of ure1 completely blocked the urea-induced trap formation in A. oligospora. However, ammonia rescued trap formation in the Aoutp79 and AoDure1 mutants. (e) Application of ammonia induced trap formation (measured at $72 \mathrm{~h}$ ). Data are expressed as mean \pm s.d. of three independent experiments. ${ }^{\star} P<0.05$, and ${ }^{\star \star \star} P<0.001$ versus WT (t-test). 
to urea, trap formation in A. oligospora responded to ammonia in a dose-dependent manner, reaching maximum induction of trap formation at $250 \mu \mathrm{g}$, whereas at $625-\mu \mathrm{g}$ concentration, ammonia suppressed the trap formation (Fig. 3e). Furthermore, the

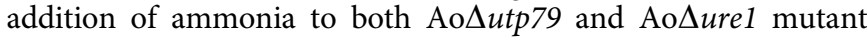
strains restored their trap formation (Fig. 3d), indicating that the urea breakdown product ammonia is the signal to induce trap formation.

Urea promotes the pathogenicity of $A$. oligospora. It has been reported that ascarosides secreted by nematodes can induce trap formation in nematode-trapping fungi ${ }^{20}$. We asked whether the bacteria/urea- and the nematode/ascaroside-induced traps share the same pathway in A. oligospora. To answer this question, we determined whether nematode induces trap formation in the AoDure 1 mutant. We found that the number of traps in the Ao $\Delta$ ure 1 mutant was comparable to that in wild-type strain in the presence of nematodes (Fig. 4a).

Next, we determined the effect of urea on nematode-induced trap formation. Nematodes and urea $\left(100 \mathrm{mgl}^{-1}\right)$ induced trap formation in A. oligospora at 16,20 and $48 \mathrm{~h}$, respectively (Fig. 4b). Although the addition of urea did not shorten the initiation time of trap formation, it significantly increased the number of traps induced by nematodes (Fig. 4b). Urea and nematodes together led to a threefold increase in the number of traps, compared with that of urea or nematodes alone. Thus, nematode and urea exhibited synergistic effect on trap formation. Meanwhile, we found that urea markedly accelerated the elimination of nematodes mediated by A. oligospora (Fig. 4c). These results suggest that the bacteria/urea signalling induces trap formation via a mechanism different from the nematode/ ascaroside signalling pathway.

Tripartite relationships in soil. In order to investigate the relationship among bacteria, nematodes and nematode-trapping fungi in the soil, we first determined the reproduction of worms in four groups containing S. maltophilia CD52, $\mathrm{Sm} \Delta$ arcA mutant,
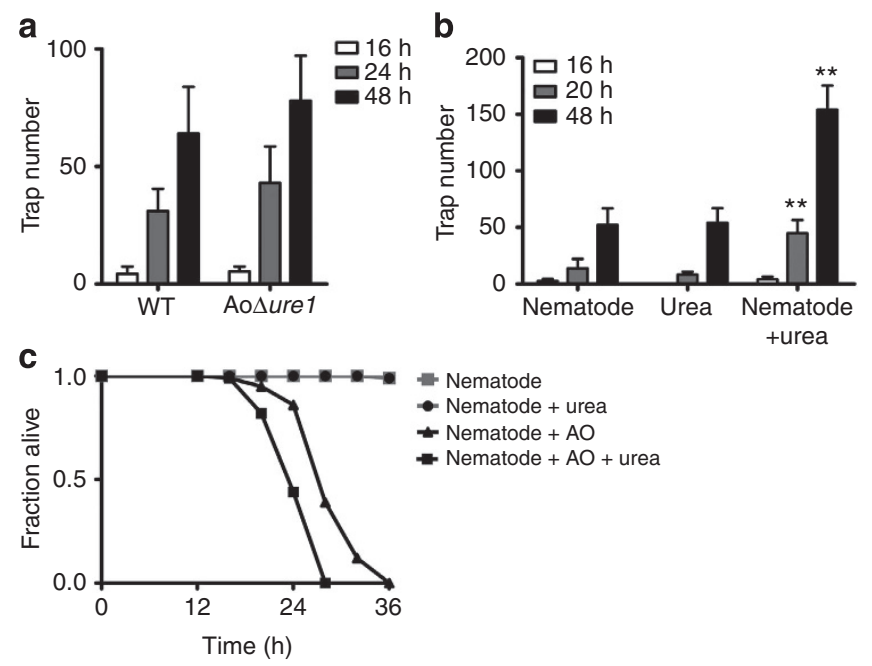

Figure 4 | Urea reduces the survival of nematodes in the presence of $\boldsymbol{A}$. oligospora. (a) The number of traps in WT and AoDure1 mutant strains in the presence of nematodes. Data are expressed as mean \pm s.d. of three independent experiments. (b) Urea promoted nematode-induced trap formation in WT strain. Data are expressed as mean \pm s.d. of three independent experiments. ${ }^{\star \star} P<0.01$ versus control (without urea; $t$-test). (c) Urea reduced the survival of nematodes after $A$. oligospora $(\mathrm{AO})$ infection. ${ }^{\star \star} P<0.01$ versus $\mathrm{AO}$ without urea ( $t$-test).
B. amyloliquefaciens $\mathrm{CD} 1$ and $\mathrm{CD} 1$ plus urea $(5 \mathrm{mg})$, respectively. B. amyloliquefaciens $\mathrm{CD1}$ is a non-urea-producing bacterium isolated from cow dung (Supplementary Table 1). As expected, the number of nematodes was all similarly increased at 72 and $96 \mathrm{~h}$ in the absence of A. oligospora (Fig. 5a). Next, we determined the effect of these bacteria on A. oligospora-nematode interaction. In the presence of A. oligospora, the soil pre-inoculated with $\mathrm{CD} 1$ plus urea or $\mathrm{CD} 52$, the number of worms started to decrease significantly at $72 \mathrm{~h}$ and almost all worms died at $96 \mathrm{~h}$ (Fig. 5b). In contrast, in soil containing $\mathrm{Sm} \Delta \operatorname{arcA}$ mutant and A. oligospora, the number of nematodes increased by 1.8 -fold at $72 \mathrm{~h}$ and began to decrease at $96 \mathrm{~h}$. Similar results were obtained in soil preinoculated with CD1 and A. oligospora (Fig. 5b). Together, our results indicate that urea-producing bacteria promote the elimination of nematodes by A. oligospora.

Next, we determined the colony-forming units of bacteria in soil. We found that all four groups exhibited a comparable number of bacteria in the presence of worms alone (Fig. 5c). The number of CD52 or CD1 (CD1 plus urea group) was much higher than that of Sm $\triangle \operatorname{arcA}$ mutant or CD1 (CD1 alone group) in the presence of worms and A. oligospora (Fig. 5d). As it is difficult to calculate the number of traps in soil, we performed similar experiments in agar plates. In the absence of nematodes, the number of traps in plates containing CD52 or CD1 plus urea increased gradually, and reached the highest level at $72 \mathrm{~h}$ (Fig. 5e). Although traps were induced by the Sm $\triangle a r c A$ mutant, the number of traps was significantly lower than that induced by CD52 or CD1 plus urea. In contrast, CD1 did not induce trap formation. Meanwhile, in the presence of worms, the number of traps in plates containing either CD52 or CD1 plus urea was significantly higher than that in plates containing $\operatorname{Sm} \Delta \operatorname{arc} A$ mutant or CD1 without urea (Fig. 5f). Taken together, our results indicate that urea-producing bacteria mobilize the nematodetrapping fungus $A$. oligospora and provide a protection against their nematode predators.

Urea diffuses readily in soil. It has been shown that the volatiles released by plants can attract insect predators from long distances $^{28-31}$. In contrast, the nonvolatile urea is likely to function as a short-range signal molecule. To examine whether urea diffuses in moist sand, $5 \mathrm{mg}$ of urea was added into a spot in a sand-filled dish. Every $30 \mathrm{~min}$, samples were taken at a distance of $8 \mathrm{~cm}$ from this spot by a small hole puncher and analysed by liquid chromatography-mass spectrometry (LC-MS). Urea was detected at the first $30 \mathrm{~min}$ after introduction in sand. The amount of urea attained the highest level at $120 \mathrm{~min}$, and declined to the basal level at $180 \mathrm{~min}$ (Supplementary Fig. 10). The rapid diffusion of urea in moist sand seems to make it suitable as a ground signal.

\section{Discussion}

As major components of the soil ecosystem, bacterivorous nematodes and bacteria interact with each other and have evolved a diversity of mechanisms to counter balance each other ${ }^{1-3}$. However, little is known about the mechanisms by which the nonpathogenic bacteria protect themselves against the nematode grazers. Our results demonstrate that certain bacteria can mobilize nematode-trapping fungi to reduce predation pressure by producing and releasing urea. The urea metabolite ammonia functions as a signal molecule to accelerate the switch in nematode-trapping fungi from saprophytic to predacious lifestyle, which is crucial for capturing nematodes (Fig. 6).

An indirect defense mechanism against predators has been reported in plants ${ }^{28-31}$ and insects ${ }^{32,33}$. When bitten by 
a

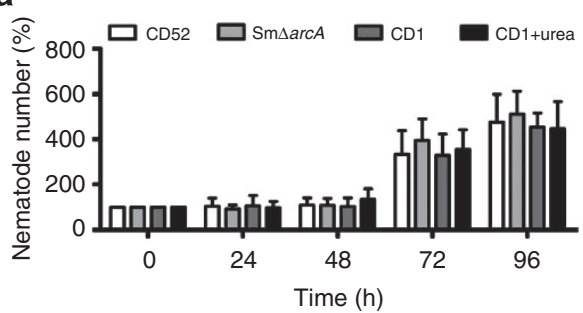

C

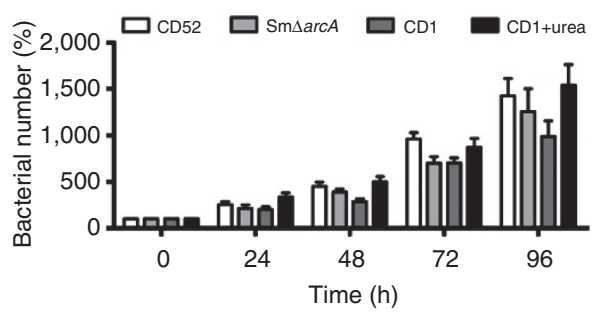

e

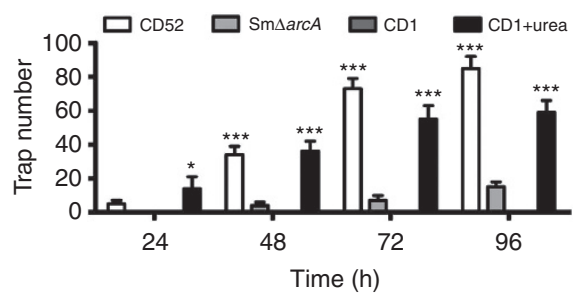

b

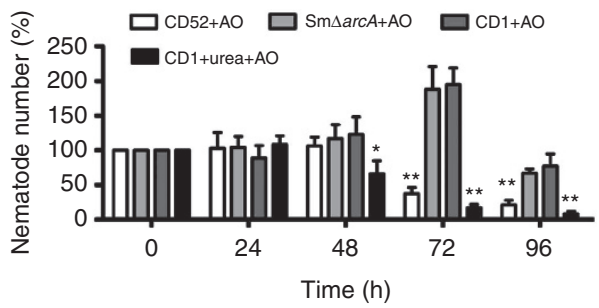

d

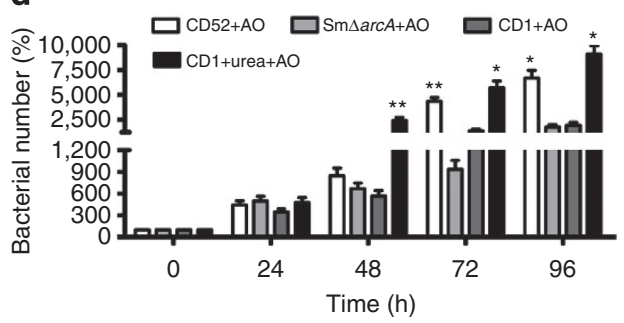

$\mathbf{f}$

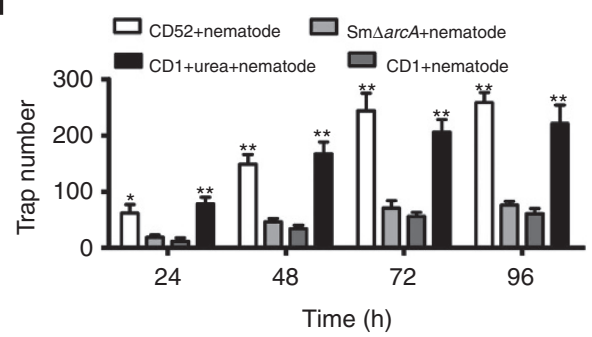

Figure 5 | The relationship among bacteria, nematodes and fungi in the soil. (a) In soil without $A$. oligospora, the number of worms steadily increased in the presence of S. maltophila CD52, Sm $\Delta$ arcA mutant, non-urea-producing bacterium B. amyloliquefaciens CD1 and CD1 plus urea (5 mg). (b) In soil with $A$. oligospora, the survival rates of worms were significantly lower in the presence of $S$. maltophila CD52 and CD1 plus urea than those in the presence of $\mathrm{Sm} \Delta \operatorname{arc} A$ mutant or B. amyloliquefaciens CD1. The data are expressed as percent change from control $(0 \mathrm{~h})$. (c) In soil without $A$. oligospora, all the four groups exhibited a comparable number of bacteria in the presence of worms. (d) In soil with A. oligospora, the number of CD52 and CD1 (CD1 plus urea group) was much higher than that of Sm $\triangle$ arcA mutant and CD1 (CD1 alone group) in the presence of worms. (e,f) The number of traps in plates containing CD52 and CD1 plus urea was significantly higher than that in plates containing Sm 4 arcA mutant and CD1 in the absence (e) or presence (f) of worms. ${ }^{\star} P<0.05,{ }^{\star \star} P<0.01$ and ${ }^{\star \star \star} P<0.001, C D 52$ versus $S m \Delta \operatorname{arc} A$ or $C D 1+$ urea versus $C D 1$ ( $t$-test).

herbivorous insects, the insect-damaged leaves and roots of plants release a large amount of volatile chemicals. These chemicals attract both parasitic and predatory insects, which are natural enemies of the herbivores, thus enabling them to locate and attack the herbivores ${ }^{28}$. Similarly, the termite (Coptotermes formosanus) uses its faeces to build its nest structure that provide nutrition to support the growth of a Streptomyces bacterium ${ }^{32}$. The presence of Streptomyces in turn inhibits the growth of Metarhizium anisopliae, an entomopathogenic fungus, providing a significant survival benefit to the termites. Thus, such multispecies interactions are likely very common in natural ecosystems.

Unlike the volatiles released by plants to attract carnivores from long distances ${ }^{28-31}$, the bacteria release nonvolatile urea as an intermediate message to communicate with fungi nearby. The high solubility and rapid diffusion of urea make it a suitable molecular signal in the ground. As urea transport is critical for inducing trap formation in A. oligospora, urea appears to function intracellularly. After entering cells, urea is metabolized to carbon dioxide and ammonia by urease. Our finding reveals that ammonia, not carbon dioxide, from urea metabolism, functions as the downstream molecule to initiate the lifestyle switch in nematode-trapping fungi. Accumulating evidence has demonstrated that both ammonia and carbon dioxide can be used as signal molecules in morphogenetic transitions in the fungal kingdom. For instance, ammonia acts as a signal to promote the reprogramming of cellular metabolism, colony differentiation and the switch from pseudohyphal to yeast-like forms in yeast species $S$. cerevisiae and C. mogii ${ }^{34,35}$. Meanwhile, carbon dioxide derived from urea breakdown is known to mediate the switch from yeast to hypha form in C. albicans inside macrophages 26 , and to induce capsule production in $C$. neoformans ${ }^{27}$. Carbon dioxide triggers the yeast-to-hypha switch of $C$. albicans by activating the cAMP-dependent protein kinase A pathway ${ }^{26}$. At present, the molecular mechanisms by which ammonia controls these fungal morphogenetic transitions remain to be investigated.

On the basis of genomic and proteomic analyses, we have previously proposed a model for trap formation induced by nematodes in the nematode-trapping fungus $A$. oligospora. According to this model, multiple signal transduction pathways are activated by nematodes, involving a variety of cellular processes such as energy metabolism, biosynthesis of the cell wall, cell division, glycerol accumulation and peroxisome biogenesis. Recently, Hsueh et al. ${ }^{20}$ have identified that ascarosides produced by $C$. elegans are responsible for inducing trap formation in nematode-trapping fungi. However, our results demonstrate that nematodes can induce trap formation in the Aosure1 mutant, implicating that nematode-mediated trap 


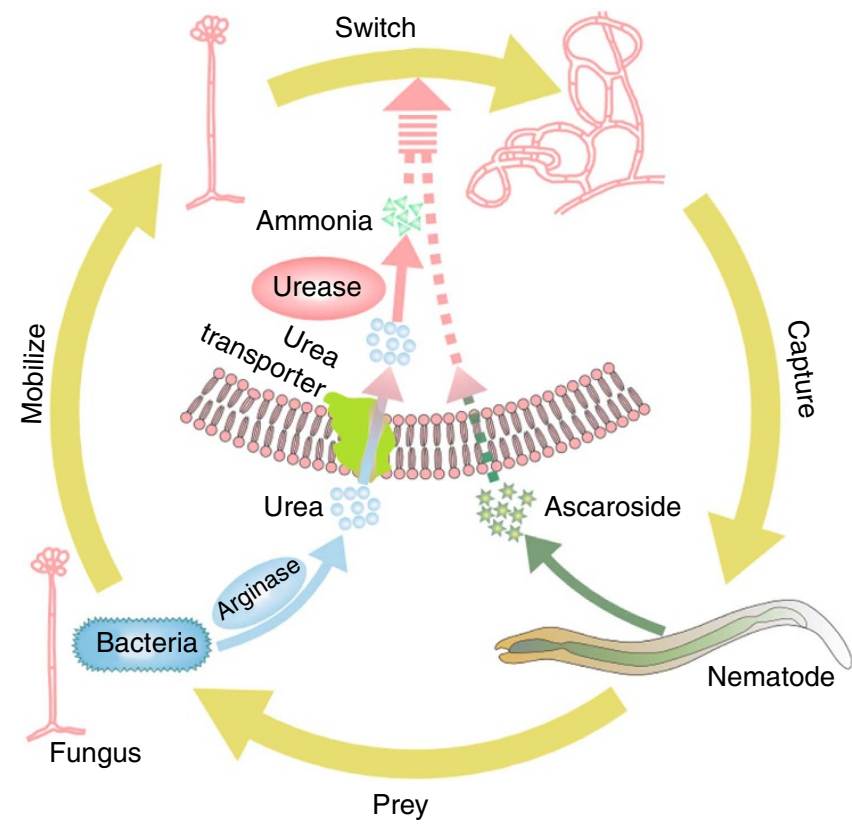

Figure 6 | A model for trap formation by nematodes and bacteria. When grazed by nematodes, bacteria increase the production and release of urea by upregulating the expression of arginase. Secreted urea is then taken up by the mycelia of nematode-trapping fungi nearby via a urea transporter, and eventually catabolized to ammonia by urease within the fungi.

Ammonia in turn initiates the lifestyle switch to form trap structures. Meanwhile, ascarosides released by nematodes are transported into the mycelia of nematode-trapping fungi by unknown proteins, and induce trap formation directly. The bacteria/urea- and the nematode/ascarosidemediated signalling pathways exhibit significant synergistic effects on trap formation, resulting in the eventual capture and death of the nematodes.

formation is independent of ammonia. Thus, the bacteria/ureaand the nematode/ascaroside-mediated signalling pathways use different mechanisms to initiate the lifestyle switch in nematodetrapping fungi. Interestingly, the application of urea is sufficient to increase the number of traps induced by nematodes, suggesting that significant synergistic effects on trap formation exist between the two signalling pathways (Fig. 6). Furthermore, the bacteria/ urea signalling markedly elicits the elimination of nematodes by A. oligospora in soil. These results suggest that these bacteria and nematode-trapping fungi form mutually beneficial relationships, thereby turning the nematodes from a bacteria hunter into a victim. The role reversals in multiple predator-prey interactions provide novel insights and add complexities to our understanding of the composition and population dynamics of microbial communities in natural ecosystems.

\footnotetext{
Methods

Induction of trap formation by cow dung. Fresh cow dung was collected from a cattle farm in Yunnan Agricultural University, Kunming, China. A. oligospora Fres. (ATCC24927) was obtained from American Type Culture Collection (ATCC). The induction of trap formation in A. oligospora by fresh or autoclaved dung was tested according to the method described previously ${ }^{21}$, with some modifications. Briefly, the cow dung $(5 \mathrm{~g})$ was diluted with $5 \mathrm{ml}$ of water and placed on a water agar

$\sim 2.5 \mathrm{~cm}$ from the rim of a $90-\mathrm{mm}$-diameter plate. After pre-incubation for 7 days at $25^{\circ} \mathrm{C}, 200 \mu \mathrm{l}$ of conidial suspension with a concentration of $\sim 5 \times 10^{4}$ conidia per $\mathrm{ml}$ was spread over the plate and incubated at $25^{\circ} \mathrm{C}$. The traps were observed and scored using a light microscope.
}

Induction of trap formation by bacteria. A total of $5 \mathrm{~g}$ fresh dung sample was added to $50 \mathrm{ml}$ of sterile water and mixed thoroughly. The aqueous suspension of fresh dung was serially diluted by 10 -folds in sterilized water and spread over LB agar plates. After 1 day of incubation at $30^{\circ} \mathrm{C}$, different colonies, named CD1 to
CD126, were picked to obtain pure cultures. These colonies were cultured in $50 \mathrm{ml}$ $\mathrm{LB}$ medium at $30^{\circ} \mathrm{C}$ for 4 days and fermentation supernatants were collected. Two hundred microlitres of each supernatant mixed with fifty microlitres conidial suspension of $A$. oligospora with a concentration of $\sim 5 \times 10^{4}$ conidia per $\mathrm{ml}$ was spread over a water agar plate and incubated at $25^{\circ} \mathrm{C}$ for $72 \mathrm{~h}$. Trap numbers were counted in 10 low-power light microscope fields and reported as the average number per field of observation.

Identification of bacteria isolated from cow dung. Bacterial genomic DNA was isolated using a commercial DNA isolation kit (Bioteke, Beijing, China). For identification of bacteria, the 16S rRNA gene sequences were amplified with the universal bacterial primers $8 \mathrm{~F}\left(5^{\prime}\right.$-AGAGTTTGATCCTGGCTCAG- $\left.3^{\prime}\right)$ and 1492R ( $5^{\prime}$-GGTTACCTTGTTACGACTT-3'). PCR products were purified using the Gel Extraction \& PCR Purification Combo Kit (Bioteke). The 16S rRNA genes were sequenced and analysed using the BLAST algorithm (http://blast.ncbi.nlm.nih.gov/ Blast.cgi).

Isolation and identification of the active compound. After S. maltophilia CD52 was grown in $5 \mathrm{l}$ of LB medium on a rotary shaker at $30^{\circ} \mathrm{C}$ for 4 days, fermentation supernatant was collected and concentrated to final volume of 11 . The supernatant was then extracted with equal volume of $n$-butanol. The $n$-butanol extract was concentrated to dryness in a rotary evaporator and the residue $(10.4 \mathrm{~g})$ was dissolved in methanol. Column chromatograph was performed on silica gel G column eluting with ethyl acetate/methanol (60:1-1:1) providing fractions $A_{1}-A_{30}$. Subsequently, these fractions were tested for their abilities to induce trap formation in A. oligospora. The active fraction $\mathrm{A}_{3}(1.62 \mathrm{~g})$ was further separated and purified on a Sephadex LH-20 column $(600 \mathrm{~g})$ eluting with methanol to yield fractions $A_{3-1}-A_{3-10}$. We found that only the fraction $A_{3-2}$ could induce trap formation. Then, the active fraction $A_{3-2}(923 \mathrm{mg})$ was again purified on Sephadex LH-20 $(200 \mathrm{~g})$ eluting with methanol to obtain a single candidate active compound (426 mg). NMR experiment was carried out on Bruker Avance III-600 NMR spectrometers with tetramethylsilane as internal standard (Bruker Corporation, Fällanden, Switzerland). Electrospray ionisation mass spectrometry profiles were recorded on a Finnigan LCQ-Advantage mass spectrometer (Thermo Electron Corp., San Jose, CA, USA). Elemental analysis was measured on VARIO ELII element analyzer (Elementar Analysensysteme GmbH, Hanau, Germany).

Detection of urea in supernatants. S. maltophilia CD 52 and $\mathrm{Sm} \Delta \operatorname{arcA}$ mutan strains were grown in $100 \mathrm{ml} \mathrm{LB}$ medium at $30^{\circ} \mathrm{C}$ for 4 days. The fermentations were extracted with equal volume of $n$-butanol. The $n$-butanol extracts were concentrated to dryness in rotary evaporator and the residues were dissolved in $10 \mathrm{ml}$ methanol. Urea was determined using LC-MS with a Waters Series HPLC 2695 (Waters Corp., Milford, MA, USA) with Thermo Finnigan LCQ Advantage mass detector (ion trap; Thermo Electron Corp.). The instrument conditions were optimized as follows: spray voltage, $5.3 \mathrm{kV}$; capillary voltage, $3.00 \mathrm{~V}$; capillary temperature, $250^{\circ} \mathrm{C}$. Samples were separated using a BDS HYPERSIL $5-\mu \mathrm{m}$ pore size $4.6 \mathrm{~mm} \times 250 \mathrm{~mm}$ column (Thermo Electron Corp.) with isocratic elution (5\% methanol) at a flow rate of $200 \mu \mathrm{min}^{-1}$. In LC-MS analysis, urea (Sigma, St Louis, MO, USA) with $\mathrm{m} / \mathrm{z} 61[\mathrm{M}+\mathrm{H}]^{+}$was used as an external standard. Retention time of urea was $15.00-16.10 \mathrm{~min}$.

Induction of trap formation by urea. For analysis of trap induction in nematodetrapping fungi by urea, $200 \mu \mathrm{l}$ of conidial suspension with a concentration of $\sim 5 \times 10^{4}$ conidia per $\mathrm{ml}$ was spread over a water agar plate. After incubating with urea $\left(0-1,500 \mathrm{mgl}^{-1}\right)$ for $72 \mathrm{~h}$, traps were counted under a light microscope. For ammonia assays, $25 \%$ of ammonia solution (Shantou Xilong Chemical, Guangdong, China) were added into one compartment of a two-compartment Petri dish with $90 \mathrm{~mm}$ diameter, and $100 \mu \mathrm{l}$ of conidial suspension with a concentration of $\sim 5 \times 10^{4}$ conidia per $\mathrm{ml}$ was spread over the other compartment containing water agar, respectively. Plates were immediately wrapped with Bemis ParafilmM sealing film (Bemis Flexible Packaging, Neenah, WI, USA) to prevent the escape of the volatiles. After incubating at $25^{\circ} \mathrm{C}$ for $72 \mathrm{~h}$, the traps were recorded under a light microscope. Three plates were performed per assay and all experiments were performed three times. Trap numbers were counted in 10 low-power light microscope fields, and reported as the average number per field of observation.

Amplification of putative arcA genes. The genomic DNA of bacteria was extracted using the DNA isolation kit (Bioteke). Total RNA from bacteria was isolated using RNAiso Plus (Takara, Dalian, China). The putative $\operatorname{arcA}$ genes were amplified from the genomic DNA and mRNA using PCR. The primers used to amplify $\operatorname{arcA}$ genes were as follows: CD1F, 5' -ACCCGCACAGGACAACATA-3', CD1R, 5'-GGAACCGCTCACTCTCAAT-3'; CD93F, 5'-CAGGAATAACATCAC CACAGT-3', CD93R, 5' -TGAAGCGGATATTGCCATAC-3'; CD102F, 5' -ATCC AAGCACTATGAAAAC-3', CD102R, 5' -GGAGAATAACCACCAATGT-3'; CD52F, 5' -TCCCATTTCCGTATCCC-3', CD52R, 5' -CCACGCCACCACTTC ATC-3'; CD82F, 5' -ACTTCCAGCCGTATGACAG-3', CD82R, 5' -ATTCACAAC CGTTCGCATAG-3'; CD101F, 5' ${ }^{\prime}$-ATCTCGGCGTCATCTGGTA-3', CD101R, $5^{\prime}$-CGTCGTTCGGATCAAGTCC- $3^{\prime}$. The primers for $16 \mathrm{~S}$ rRNA gene were as 
follows: 533F, 5'-GTGCCAGCAGCCGCGGTAA-3', 907R, 5'-CCGTCAATTCM TTTRAGTTT- $3^{\prime}$.

Deletion of arcA in S. maltophilia. The S. maltophilia CD52 arcA knockout vector was constructed by using the gene replacement vector pSUP202. Briefly, a 925-bp fragment spanning the entire coding region of the $\operatorname{arcA}$ gene, was amplified from genomic DNA of S. maltophilia CD52 strain by PCR using primers arcAF (5'-GAA TTCATGGCCCATCCCATCTCCGTATC- $3^{\prime}$ ) containing a BamHI endonuclease recognition site and arcAR (5'-AAGCTTGCATCAGCGTGGACTTGCCGAAC-3') containing a HindIII recognition site. The fragment was inserted into pMD-18T vector by T/A cloning, resulting in $\mathrm{pMD}$-arcA. A 1,614-bp fragment of tetracycline $(\mathrm{Tc})$ amplified from plasmid pRK415 with primers $415 \mathrm{TcF}$ (5'-CTGCAGAGTTT GCGTGTCGTCAGAC- $3^{\prime}$ ) containing a PstI recognition site and 415TcR (5'-GCA TGCTCCTTACTGGGCTTTCTCA- $3^{\prime}$ ) containing a $S p h \mathrm{I}$ recognition site. The fragment was inserted into pMD-18T vector by T/A cloning, resulting in pMD-Tc. The plasmids of $\mathrm{pMD}$-arcA and $\mathrm{pMD}-\mathrm{Tc}$ were digested with $P s t \mathrm{I} / \mathrm{Sph \textrm {I }}$, respectively. The Tc PstI/SphI fragment was cloned into the PstI/SphI gaped site of $\operatorname{arcA}$, resulting in the $\mathrm{pMD}$-arcA-Tc vector. Considering the size of the $\operatorname{arcA}-\mathrm{Tc}$ fragment is close to that of the pMD-18T vector, the plasmid pMD-arcA-Tc was digested with DraI. The fragment was then digested with BamHI/HindIII and cloned into pSUP202. The final plasmid pSUP-arcA-Tc was introduced into Escherichia coli S17-1 ( $\lambda$ pir) using an electroporation programme with a pulse charge at $2.5 \mathrm{kV}$ and pulse time of 6 ms. The plasmid was then mobilized into S. maltophilia CD52 via conjugation.

Transconjugants were performed on LB agar supplemented with $50 \mu \mathrm{g} \mathrm{ml}^{-1}$ of tetracycline, $100 \mu \mathrm{g} \mathrm{ml}^{-1}$ of ampicillin and $50 \mu \mathrm{g} \mathrm{ml}^{-1}$ of kanamycin. The resistant colonies were selected into LB broth supplemented with $50 \mu \mathrm{g} \mathrm{ml}^{-1}$ of tetracycline and then incubated overnight at $30^{\circ} \mathrm{C}$. DNA from tetracyclineresistant colonies were isolated and confirmed using PCR. The primers used for PCR were arcAF and arcAR.

Quantitative real-time PCR analysis. Total RNA from S. maltophilia CD52 was isolated using RNAiso Plus (Takara). Reverse transcription was conducted based on RNA extracted using PrimeScript RT reagent Kit with gDNA Eraser (Perfect Real Time; Takara) according to the manufacturer's instructions. A real time-PCR analysis was performed with the Roche LightCycler 480 System (Roche Applied Science, Penzberg, Germany) using SYBR Premix-Ex Tag GC (Takara). The primers used for PCR were as follows: $\operatorname{arcA}: 5^{\prime}-$ GTCGCTGGACATCGTTGAG-3' (F), $5^{\prime}$-CGTGGACTTGCCGAACAG-3' (R); rpoD: $5^{\prime}$-GGGCGAAGAAGGAAATG GTC-3' (F), 5'-CAGGTGGCGTAGGTGGAGAA-3' (R).

Deletion of genes in A. oligospora. To delete utp79, two fragments (1,500 and $1,583 \mathrm{bp}$ ) were amplified with PCR using utp79-5F/utp79-5R and utp79-3F/utp79$3 \mathrm{R}$, respectively. For deletion of $u t p 215$, two fragments (both of $1,674 \mathrm{bp}$ ) were amplified with PCR using utp215-5F/utp215-5R and utp215-3F/utp215-3R, respectively. For deletion of ure1, two fragments (both of 2,387 bp) were amplified with PCR using ure1-5F/ure1-5R and ure1-3F/ure1-3R. The sequences of these primers were as follows: utp79-5F, 5'-GTAACGCCAGGGTTTTCCCAGTCACGA CGCAAAGCCGTTTATCAAGAAC-3' ${ }^{\prime}$ utp79-5R, 5' -ATCCACTTAACGTTAC TGAAATCTCCAACAGAATACCAGAACCGAGACC-3'; utp79-3F, 5' -CTCCTT CAATATCATCTTCTGTCTCCGACGTCAACTCCGTTATCAATCT-3' ${ }^{\prime}$, utp793R, 5'-CGGATAACAATTTCACACAGGAAACAGCCATCCATCTAACCCCTC TTT-3'; utp215-5F, 5'-GTAACGCCAGGGTTTTCCCAGTCACGACGCGGTTGG CAAAGATAAGCAG-3' ${ }^{\prime}$, utp215-5R, 5' -ATCCACTTAACGTTACTGAAATCTC CAACAGGCGGAGTGAGTGTAGTCG-3'; utp215-3F, $5^{\prime}$-CTCCTTCAATATCA TCTTCTGTCTCCGACGATGTTTCGGCTGGTCTCGT-3' ${ }^{\prime}$, utp215-3R, 5' -GCGG ATAACAATTTCACACAGGAAACAGCTGTTGGATTTTGGGATAGGT-3'; ure1-5F, 5'-GTAACGCCAGGGTTTTCCCAGTCACGACGCATTATCGTCTCA ACTACCC-3' ${ }^{\prime}$, ure1-5R, 5' -ATCCACTTAACGTTACTGAAATCTCCAACTATT CATTTTATTTGTCCGC-3'; ure1-3F, $5^{\prime}$-CTCCTTCAATATCATCTTCTGTCTC CGACTTGTGGGTTGGTTTCTATG- ${ }^{\prime}$, ure1-3R, $5^{\prime}$-GCGGATAACAATTTCAC ACAGGAAACAGCTGCTCTTTTCTTGCTTTCT- $3^{\prime}$. The deletion cassettes were constructed by double crossing-over homologous recombination according to the method described previously 36,37 . The primers used for PCR were as follows: utp79F, 5'-CAGGAACCAATCCAAAGAGC-3' ${ }^{\prime}$, utp79R, 5' -CAACCCAATAAAG TCGCACG-3'; utp215F, 5'-GCTGTGGTTCACTACCTTAC-3' ${ }^{\prime}$, utp215R, 5' -ACA CCCATCATCAAGTAGAG-3'; ure1F, 5'-AGTCGTAGCATTCGTCCCAG-3', ure1R, 5' $5^{\prime}$-ACCGTTCATCACCCCATTTC-3'.

Uptake of $\left[{ }^{13} \mathbf{C}_{1}{ }^{15} \mathbf{N}_{\mathbf{2}}\right]$-urea. About $200 \mu \mathrm{l}$ of conidial suspension of A. oligospora with a concentration of $\sim 1 \times 10^{6}$ conidia per $\mathrm{ml}$ was grown in potato dextrose broth medium. After 6 days, the mycelia were washed and re-suspended in water. $\left[{ }^{13} \mathrm{C}^{15} \mathrm{~N}_{2}\right]$-Urea uptake was determined using MM containing $0.2 \mathrm{mg} \mathrm{ml}^{-1}$ $\left[{ }^{13} \mathrm{C},{ }^{15} \mathrm{~N}_{2}\right]$-urea (Sigma) at $25^{\circ} \mathrm{C}$, pH 6.8. After incubation for 30 and $120 \mathrm{~min}$, mycelium was collected. The resulting mycelial pellet $(100 \mathrm{mg})$ was washed with PBS five times. The pellet was ground with liquid nitrogen and extracted using $2 \mathrm{ml}$ methanol. The extracts were then condensed to $100 \mu \mathrm{l}$. $\left[{ }^{13} \mathrm{C},{ }^{15} \mathrm{~N}_{2}\right]$-Urea was determined with LC-MS. For LC-MS quantification, $\left[{ }^{13} \mathrm{C},{ }^{15} \mathrm{~N}_{2}\right]$-urea with $m / z 64[\mathrm{M}+\mathrm{H}]^{+}$was used as an external standard. The retention time of $\left[{ }^{13} \mathrm{C},{ }^{15} \mathrm{~N}_{2}\right]$-urea was $15.00-16.10 \mathrm{~min}$. A calibration curve was constructed using known concentrations of urea ranging from $200 \mathrm{ng} \mathrm{ml}^{-1}$ to $10 \mathrm{mg} \mathrm{ml}^{-1}$. Each solution of $10 \mu \mathrm{l}(2-100 \mathrm{ng})$ was injected on the column. All assays were performed in duplicates. Experimental data were calibrated and quantified using the Xcalibur Software (Thermo Finnigan). The calibration curves were constructed based on peak areas of the calibrated standards. Concentrations of all analytes were calculated from their peak areas against the calibration curves. The limit of detection for each analyte was determined as three times the signal-to-noise ratio. Recovery percent for each analyte in each matrix was obtained as the calculated concentration divided by the actual concentration.

Induction of trap formation by nematodes. For analysis of trap formation induced by the nematode C. elegans, $200 \mu \mathrm{l}$ of conidial suspension with a concentration of $\sim 5 \times 10^{4}$ conidia per ml was inoculated on water agar. After 2 days of growth, 80-100 adult nematodes were added to A. oligospora mycelia in the presence or absence of urea $\left(100 \mathrm{mgl}^{-1}\right)$. After $16 \mathrm{~h}$, mycelia were observed at time intervals under light microscope (Olympus, Tokyo, Japan).

Interaction among bacteria, nematodes and fungi. Plates $(35 \mathrm{~mm})$ were first filled with sterilized soil $(3 \mathrm{~g})$. Bacterial suspension $(500 \mu \mathrm{l}$; OD600 $=0.1), \sim 300$ synchronized L3 C. elegans larvae or $100 \mu \mathrm{l}$ of urea $\left(50 \mathrm{mg} \mathrm{ml}^{-1}\right)$, was added to each plate in the presence or absence of $200 \mu \mathrm{l}$ conidia of A. oligospora $\left(5 \times 10^{4}\right.$ conidia per ml). After inoculation for $24 \mathrm{~h}$ at $25^{\circ} \mathrm{C}$, the soil samples were collected at $24-\mathrm{h}$ intervals. The soil samples were suspended in $10-\mathrm{ml}$ sterile water. To count worm numbers, $1 \mathrm{ml}$ of suspension of each treatment was taken and the number of worms was counted under a light microscope. To count colony-forming unit of bacteria, the suspension of each treatment was serially diluted by 10 -folds in sterilized water and spread over LB agar plates. After 1 day of incubation at $30^{\circ} \mathrm{C}$ bacterial colonies were counted.

For measurement of traps in the presence of nematodes and bacteria, $200 \mu \mathrm{l}$ conidia of A. oligospora $\left(5 \times 10^{4}\right.$ conidia per $\left.\mathrm{ml}\right)$ were pre-inoculated in water agar plates for $36 \mathrm{~h}$. Then, $200 \mu \mathrm{l}$ bacterial suspension $(\mathrm{OD} 600=0.1)$ or $100 \mu \mathrm{l}$ of urea $\left(50 \mathrm{mg} \mathrm{ml}^{-1}\right)$ with and without 60 synchronized L3 C. elegans larvae were added to water agar plates. After inoculation at $25^{\circ} \mathrm{C}$ for $24 \mathrm{~h}$, the traps were observed and scored using a light microscope at the indicated time points.

Urea diffusion measurements. The diffusion of urea was determined by the method described by Rasmann et al. ${ }^{30}$, with some modifications. Briefly, a glass dish $(30 \mathrm{~cm}$ in diameter, $8 \mathrm{~cm}$ deep) was filled with a $3-\mathrm{cm}$ layer of moist sand $(10 \%$ water). With a micropipette, $5 \mathrm{mg}$ of urea in $100 \mu \mathrm{l}$ of water was placed $1 \mathrm{~cm}$ deep in the sand at the centre of the dish. At a distance of $8 \mathrm{~cm}$ from this spot, the samples were taken by a small hole puncher $(0.8 \mathrm{~cm}$ in diameter and $1 \mathrm{~cm}$ deep) every $30 \mathrm{~min}$. The urea in the sand was analysed with LC-MS.

Statistics. Differences in gene expression, trap formation, urea content and nematode number were assessed by the Student's $t$-test. Data were analysed using the SPSS11.0 software (SPSS Inc.).

\section{References}

1. De Mesel, I. et al. Top-down impact of bacterivorous nematodes on the bacterial community structure: a microcosm study. Environ. Microbiol. 6, 733-744 (2004).

2. Postma-Blaauw, M. B. et al. Within-trophic group interactions of bacterivorous nematode species and their effects on the bacterial community and nitrogen mineralization. Oecologia 142, 428-439 (2005).

3. Jousset, A. Ecological and evolutive implications of bacterial defences against predators. Environ. Microbiol. 14, 1830-1843 (2012).

4. Jousset, A. et al. Predators promote defence of rhizosphere bacterial populations by selective feeding on non-toxic cheaters. ISME J. 3, 666-674 (2009).

5. Gallagher, L. A. \& Manoil, C. Pseudomonas aeruginosa PAO1 kills Caenorhabditis elegans by cyanide poisoning. J. Bacteriol. 183, 6207-6214 (2001).

6. Mahajan-Miklos, S., Tan, M. W., Rahme, L. G. \& Ausubel, F. M. Molecular mechanisms of bacterial virulence elucidated using a Pseudomonas aeruginosaCaenorhabditis elegans pathogenesis model. Cell 96, 47-56 (1999).

7. Vaitkevicius, K. et al. A Vibrio cholerae protease needed for killing of Caenorhabditis elegans has a role in protection from natural predator grazing. Proc. Natl Acad. Sci. USA 103, 9280-9285 (2006).

8. Niu, Q. et al. A Trojan horse mechanism of bacterial pathogenesis against nematodes. Proc. Natl Acad. Sci. USA 107, 16631-16636 (2010).

9. Darby, C., Hsu, J. W., Ghori, N. \& Falkow, S. Caenorhabditis elegans: plague bacteria biofilm blocks food intake. Nature 417, 243-244 (2002).

10. Tan, L. \& Darby, C. A movable surface: formation of Yersinia sp. biofilms on motile Caenorhabditis elegans. J. Bacteriol. 186, 5087-5092 (2004).

11. Shtonda, B. B. \& Avery, L. Dietary choice behavior in Caenorhabditis elegans. J. Exp. Biol. 209, 89-102 (2006). 
12. Schulenburg, H. \& Ewbank, J. J. The genetics of pathogen avoidance in Caenorhabditis elegans. Mol. Microbiol. 66, 563-570 (2007).

13. Pradel, E. et al. Detection and avoidance of a natural product from the pathogenic bacterium Serratia marcescens by Caenorhabditis elegans. Proc. Natl Acad. Sci. USA 104, 2295-2300 (2007).

14. Shivers, R. P., Kooistra, T., Chu, S. W., Pagano, D. J. \& Kim, D. H. Tissuespecific activities of an immune signaling module regulate physiological responses to pathogenic and nutritional bacteria in C. elegans. Cell Host Microbe 6, 321-330 (2009).

15. Zhang, Y., Lu, H. \& Bargmann, C. I. Pathogenic bacteria induce aversive olfactory learning in Caenorhabditis elegans. Nature 438, 179-184 (2005).

16. Barron, G. L. in Topics in Mycobiology No. 1 (Canadian Biological Publications, 1977).

17. Yang, J. et al. Genomic and proteomic analyses of the fungus Arthrobotrys oligospora provide insights into nematode-trap formation. PLoS Pathog. 7, e1002179 (2011)

18. Pramer, D. \& Stoll, N. R. Nemin: a morphogenic substance causing trap formation by predaceous fungi. Science 129, 966-967 (1959).

19. Choe, A. et al. Ascaroside signaling is widely conserved among nematodes. Curr. Biol. 22, 772-780 (2012)

20. Hsueh, Y. P., Mahanti, P., Schroeder, F. C. \& Sternberg, P. W. Nematodetrapping fungi eavesdrop on nematode pheromones. Curr. Biol. 23, 83-86 (2013).

21. Dackman, C. \& Nordbring-Hertz, B. Conidial traps-a new survival structure of the nematodetrapping fungus Arthrobofrys oligospora. Mycol. Res. 96, 194-198 (1992).

22. Perozich, J., Hempel, J. \& Morris, Jr S. M. Roles of conserved residues in the arginase family. Biochim. Biophys. Acta 1382, 23-37 (1998).

23. ElBerry, H. M., Majumdar, M. L., Cunningham, T. S., Sumrada, R. A. \& Cooper, T. G. Regulation of the urea active transporter gene (DUR3) in Saccharomyces cerevisiae. J. Bacteriol. 175, 4688-4698 (1993).

24. Morel, M. et al. Characterization and regulation of PiDur3, a permease involved in the acquisition of urea by the ectomycorrhizal fungus Paxillus involutus. Fungal Genet. Biol. 45, 912-921 (2008).

25. Navarathna, D. H., Das, A., Morschhauser, J., Nickerson, K. W. \& Roberts, D. D. Dur3 is the major urea transporter in Candida albicans and is co-regulated with the urea amidolyase Dur1,2. Microbiology 157, 270-279 (2011).

26. Ghosh, S. et al. Arginine-induced germ tube formation in Candida albicans is essential for escape from murine macrophage line RAW 264.7. Infect Immun. 77, 1596-1605 (2009).

27. Frazzitta, A. E. et al. Nitrogen source-dependent capsule induction in humanpathogenic cryptococcus species. Eukaryot. Cell 12, 1439-1450 (2013).

28. Pare, P. W. \& Tumlinson, J. H. Plant volatiles as a defense against insect herbivores. Plant Physiol. 121, 325-332 (1999).

29. Allmann, S. \& Baldwin, I. T. Insects betray themselves in nature to predators by rapid isomerization of green leaf volatiles. Science 329, 1075-1078 (2010).

30. Rasmann, S. et al. Recruitment of entomopathogenic nematodes by insectdamaged maize roots. Nature 434, 732-737 (2005).

31. Allison, J. D. \& Daniel Hare, J. Learned and naive natural enemy responses and the interpretation of volatile organic compounds as cues or signals. New Phytol. 184, 768-782 (2009).
32. Chouvenc, T., Efstathion, C. A., Elliott, M. L. \& Su, N. Y. Extended disease resistance emerging from the faecal nest of a subterranean termite. Proc. Biol. Sci. 280, 20131885 (2013).

33. Kaltenpoth, M., Gottler, W., Herzner, G. \& Strohm, E. Symbiotic bacteria protect wasp larvae from fungal infestation. Curr. Biol. 15, 475-479 (2005).

34. Palkova, Z. et al. Ammonia mediates communication between yeast colonies. Nature 390, 532-536 (1997).

35. Palkova, Z. et al. Ammonia pulses and metabolic oscillations guide yeast colony development. Mol. Biol. Cell 13, 3901-3914 (2002).

36. Colot, H. V. et al. A high-throughput gene knockout procedure for Neurospora reveals functions for multiple transcription factors. Proc. Natl Acad. Sci. USA 103, 10352-10357 (2006).

37. Chen, Y. L., Gao, Y., Zhang, K. Q. \& Zou, C. G. Autophagy is required for trap formation in the nematode-trapping fungus Arthrobotrys oligospora. Environ Microbiol. Rep. 5, 511-517 (2013).

\section{Acknowledgements}

We are grateful to W. Xue (MIT), H. Yin (MIT) and Y. Zhang (Harvard Medical School) for their critical reading of this manuscript. We thank ATCC for A. oligospora. This work was supported in part by the grants (2013CB127500 and 2012CB722208) from the National Basic Research Programme of China (973), the grants from the National Natural Science Foundation of China (U1036602 and 31360028).

\section{Author contributions}

K.-Q.Z., C.-G.Z. and G.-H.L. designed the experiments and analysed the data. X.W., G.-H.L., T.L., P.-J.Z., L.-M.L., X.Z., Y.-K.Q., M.-Q.T. and Y.-Y.X. performed the experiments. X.-L.J., J.-P.X., Z.-Q.A., Y.-C.M., Z.-F.Y., X.-W.H., S.-Q.L., X.-M.N. and J.-K.Y. interpreted the data, Y.H. provided the key reagents. K.-Q.Z., C.-G.Z., X.W., J.-P.X. and G.-H.L. wrote the manuscript. All authors discussed the results and commented on the manuscript.

\section{Additional information}

Supplementary Information accompanies this paper at http://www.nature.com/ naturecommunications

Competing financial interests: The authors declare no competing financial interests.

Reprints and permission information is available online at http://npg.nature.com/ reprintsandpermissions/

How to cite this article: Wang, X. et al. Bacteria can mobilize nematode-trapping fungi to kill nematodes. Nat. Commun. 5:5776 doi: 10.1038/ncomms6776 (2014).

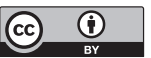

This work is licensed under a Creative Commons Attribution 4.0 International License. The images or other third party material in this article are included in the article's Creative Commons license, unless indicated otherwise in the credit line; if the material is not included under the Creative Commons license, users will need to obtain permission from the license holder to reproduce the material. To view a copy of this license, visit http://creativecommons.org/licenses/by/4.0/ 